\title{
Surgical Management of Chiari Malformations: Preliminary Results of Surgery According to the Mechanisms of Ptosis of the Brain Stem and Cerebellum
}

\author{
Misao Nishikawa ${ }^{1,2,3}$ Paolo A. Bolognese ${ }^{2,4}$ Roger W. Kula²,4 Hiromichi Ikuno ${ }^{5}$ Toshihiro Takami ${ }^{3}$ \\ Kenji Ohata ${ }^{3}$ \\ ${ }^{1}$ Department of Neurosurgery, Moriguchi-lkuno Memorial Hospital, \\ Koudoukai Health System, Osaka, Japan \\ 2 The Chiari Institute, North Shore University Hospital, New York, \\ United States \\ ${ }^{3}$ Department of Neurosurgery, Osaka City University Graduate \\ School of Medicine, Osaka, Japan \\ ${ }^{4}$ Chiari Neurosurgical Center, Long Island, New York, United State \\ ${ }^{5}$ Department of Neuroradiology, Moriguchi-lkuno Memorial Hospital, \\ Koudoukai Health System, Osaka, Japan \\ Address for correspondence Misao Nishikawa, MD, PhD, Department \\ of Neurosurgery, Moriguchi-lkuno Memorial Hospital, 6-17-33 \\ Satanakamachi, Moriguchi City, Osaka 570-0002, Japan \\ (e-mail: misaonishikawa88@gmail.com). \\ J Neurol Surg B 2021;82:264-272.
}

\begin{abstract}
Keywords

- Chiari malformation

- morphometric study

- posterior cranial fossa

- craniovertebral junction

- surgical management

- outcome
\end{abstract}

Introduction We classified Chiari malformation type I (CM-I) according to the mechanism of ptosis of the brain stem and cerebellum, based on a morphometric study of the posterior cranial fossa (PCF) and craniovertebral junction (CVJ). Surgery was performed to manage the mechanism of the hindbrain ptosis.

Materials and Methods We calculated the volume of the PCF (VPCF) and the area surrounding the foramen magnum (VSFM) and measured the axial length of the enchondral parts of the occipital bone (occipital bone size) and the hindbrain. According to these measures, we classified CM-I into type A (normal VPCF, normal VSFM, and normal occipital bone size), type B (normal VPCF, small VSFM, and small occipital bone size), and type C (small VPCF, small VSFM, and small occipital bone size). Foramen magnum decompression (FMD) (280 cases) was performed on CM-I types A and B. Expansive suboccipital cranioplasty (ESCP) was performed on CM-I type C. Posterior craniocervical fixation (CCF) was performed in cases with CVJ instability. Lysis of the adhesion and/or sectioning of the filum terminale were performed on cases with tethered cord syndrome.

Results Both ESCP and FMD had a high rate of improvement of neurological symptoms (87\%) and recovery rate. There was only small number of complications. CCF had a high rate of improvement of neurological symptoms (88\%) and joint stabilization.

Conclusion In the management of Chiari malformation, appropriate surgical methods that address ptosis of the hindbrain should be chosen. Each surgical approach resulted in a good improvement of neurological symptoms. received

June 1, 2019

accepted

August 11, 2019

published online

September 30, 2019 (c) 2019. Thieme. All rights reserved.

Georg Thieme Verlag KG,

Rüdigerstraße 14,

70469 Stuttgart, Germany
DOI https://doi.org/

10.1055/s-0039-1697977. ISSN 2193-6331. 


\section{Background and Purpose}

Previously, we reported that patients with a Chiari malformation have an underdeveloped occipital bone, so that the posterior cranial fossa (PCF) becomes shallow. ${ }^{1,2}$ This shallowness results in the brain stem and cerebellum sagging into the spinal canal, that is, the pathogenesis of Chiari malformation is insufficiency of the para-axial mesoderm, which is the origin of the occipital bone. ${ }^{1-7}$ In this case, foramen magnum decompression (FMD) to enlarge the space of the foramen magnum has been performed widely. Other mechanisms of ptosis of the brain stem and cerebellum, that is, hypermobility and instability of the craniovertebral junction (CVJ), traction by tethering, and pressure coning, have also been reported. ${ }^{8-13}$ In those cases, FMD does not resolve the underlying mechanism. Misunderstandings of the mechanism of ptosis of the brain stem and cerebellum in Chiari malformation have resulted in confusion when choosing a surgical approach, and the use of inappropriate treatment has resulted in the recurrence of neurological symptoms.

Since 2006, we have performed morphometric studies of the PCF using magnetic resonance imaging (MRI) and computed tomography (CT) reconstructed images, and have conducted multiple analyses on cases with a Chiari malformation. In addition, we have also examined the mechanism of ptosis of the brain stem and cerebellum using morphometric analysis and have redefined and classified Chiari malformation type I (CM-I) into three independent subgroups: CM-I types A, B, and C. ${ }^{14}$ We have performed surgical procedures to treat the mechanism of ptosis of the brain stem and cerebellum (-Tables 1 and 2). ${ }^{14}$ We also present the preliminary results of treatments for ptosis of the brain stem and cerebellum in cases with CM-I.

\section{Materials}

We recruited 100 healthy control volunteers with no neurological symptoms or abnormalities in the neural axis (1669 years old, mean: 38.7 years, 40 males, 60 females) under approval from the Institutional Review Boards of Koudoukai Health System (Osaka City University Graduate School of Medicine, Osaka, Japan) and North Shore University Hospital-Long Island Jewish Health System (New York, New York, United States). For the cases, 500 subjects with CM-I (cerebellar tonsil herniation $\geq 5 \mathrm{~mm}$ from the McRae line, i.e., between the basion and opisthion) (16-69 years old, mean: 37.7 years, 237 males, 263 females) and 50 subjects with an absence of CM-I (defined as cerebellar tonsil herniation $<5 \mathrm{~mm}$ from the McRae line, but having brain stem symptoms and/or myelopathy due to associated syringomyelia) (17-55 years old, mean: 35.4 years, 18 males, 32 females) were examined. The distribution and mean of age and sex were not significantly different between the normal controls and CM-I group. CM-I was associated with syringomyelia in 221 cases, hereditary disorders in connective tissue in 187 cases, basilar invagination in 23 cases, and other bony anomalies in the CVJ in 57 cases. Patients who had syringomyelia due to other etiology were excluded. These patients were diagnosed and treated from April 2006 to March 2017.
Table 1 Subtypes of CM-I classified based on morphometric analyses and surgical indication

\begin{tabular}{|l|l|l|l|l|}
\hline & $\begin{array}{l}\text { CM-I } \\
\text { type A }\end{array}$ & $\begin{array}{l}\text { CM-I } \\
\text { type B }\end{array}$ & $\begin{array}{l}\text { CM-I } \\
\text { type C }\end{array}$ & CM-absence \\
\cline { 2 - 5 } & $\begin{array}{l}187 \\
\text { cases }\end{array}$ & $\begin{array}{l}178 \\
\text { cases }\end{array}$ & $\begin{array}{l}155 \\
\text { cases }\end{array}$ & $\begin{array}{l}50 \\
\text { cases }\end{array}$ \\
\hline PCFV & Normal & Normal & Small & Normal \\
\hline VAFM & Normal & Small & Small & Small \\
\hline PFBV/PFCV & Normal & Large & Large & Normal \\
\hline $\begin{array}{l}\text { Occipital } \\
\text { bone size }\end{array}$ & Normal & Small & Small & Normal \\
\hline Surgery & $\begin{array}{l}\text { Others } \\
\text { and FMD }\end{array}$ & FMD & ESCP & FMD \\
\hline
\end{tabular}

Abbreviations: CM-absence, cases which have neurological brain stem symptoms but tonsillar herniation less than $5 \mathrm{~mm}$; CM-I, Chiari malformation type I; ESCP, expansive suboccipital cranioplasty; FMD, foramen magnum decompression; PCFV, posterior fossa cranial volume; PFBV, the volume of brain in posterior cranial fossa; VAFM, the volume of area of foramen magnum.

Table 2 Other mechanisms of ptosis of the brain stem and cerebellum, and surgical indications

\begin{tabular}{|c|c|c|c|}
\hline & CVI & $\begin{array}{l}\text { Traction } \\
\text { (tethering) }\end{array}$ & Others \\
\hline & 50 cases & 20 cases & 17 cases \\
\hline PCFV & Normal & Normal & Normal \\
\hline VAFM & Normal & Normal & Normal \\
\hline PFBV / PCFV & Normal & Normal & \\
\hline $\begin{array}{l}\text { Brain stem } \\
\text { and cerebellum }\end{array}$ & Normal & \multicolumn{2}{|c|}{$\begin{array}{l}\text { Elongation and/or } \\
\text { downward displacement }\end{array}$} \\
\hline Surgery & $\mathrm{CCF}$ & Untethering/SFT & $\begin{array}{l}\text { VPS and/ } \\
\text { or others }\end{array}$ \\
\hline
\end{tabular}

Abbreviations: CCF, craniocervical fixation; CVI, craniovertebral instability; PCFV, posterior fossa cranial volume; PFBV, the volume of brain in posterior cranial fossa; SFT, section of filum terminale; VAFM, the volume of area of foramen magnum; VPS, ventriculoperitoneal shunt.

\section{Methods: Morphometric Analyses and Surgical Indication}

\section{Morphometric Analyses and Classification of CM-I (-Table 1)}

By MRI, two-dimensional (2D) and three-dimensional CT reconstructed images using OsiriX software (free access) were used to calculate the volume of the PCF (VPCF), brain volume in the PCF (VPCB), and volume of the area surrounding the foramen magnum (VSFM). The axial length of the basiocciput, exocciput, and supraocciput of the enchondral parts of the occipital bone (occipital bone size), axial length of the brain stem, cerebellum (excluding herniated tonsils), and the position of the fourth ventricle were also measured. By multiple analyses of the results, CM-I was classified into three independent groups: CM-I types A (167 cases) (normal VPCF, normal VSFM, and normal occipital bone size), B (178 cases) (normal VPCF, small VSFM, and small occipital bone 
size), and C (155 cases) (small VPCF, small VSFM, and small occipital bone size). ${ }^{1,2,13}$

The volume of the brain stem and cerebellum and morphometric analyses (axial length of the brain stem and cerebellum) indicated there was no significant difference between the cases and healthy controls. Of the measured items, the following variables were significantly different in the cases compared with the controls: VPCF, the ratio of VPCB to VPCF (VPCB: VPCF), occipital bone size (axial length of the basiocciput and exocciput), and VSFM. In CM-I type A (167 cases), there was no significant difference in VPCF, VSFM, and occipital bone size compared with normal controls (-Table 1). In CM-I type B (178 cases), there was no significant difference in VPCF compared with normal controls, but VSFM and occipital bone size were significantly smaller than in normal controls. In this group, the volume above Twining's line (the line between the tubercle sellar and internal occipital protuberance) (VPCF-ATL) was significantly smaller than in the other groups, and the volume below Twining's line (VPCF-BTL) was also significantly smaller than in the other groups ( $\mathbf{-}$ Table $\mathbf{1}$ ). In CM-I type C ( 155 cases), VPCF, VSFM, and occipital bone size were significantly smaller than in normal controls. In this group, VPCF-ATL and VPCF-BTL were significantly smaller than in the other groups ( - Table $\mathbf{1}$ ). Moreover, the axial length of the brain stem was significantly longer than in the other groups, suggesting elongation of the brain stem in CM-I type $C$.

In CM-II (30 cases), VPCF and occipital bone size were significantly smaller than in normal controls, while the brain stem was longer, similar to the pattern observed in CM-I type C. However, VSFM was significantly larger than in normal controls (-Table 1). In addition, VSFM was significantly larger in CM-I type B than in CM-I type A.

The VSFM of patients with CM-I type A who had tethered cord syndrome was larger than in normal controls; however, VSFM was not significantly different between patients with CM-I type A who had a tumor and normal controls.

\section{Hypermobility and Instability at the Craniovertebral Junction and Tethered Cord Syndrome (- Table 2)}

Diagnosis of instability at the CVJ was confirmed by a morphometric study and a craniocervical traction test, using morphometric analyses described by the authors and Goel et al. ${ }^{11-13}$ The first author (M.N.: neurosurgeon), second author (P.B.: neurosurgeon), and fourth author (H.I.) performed the craniocervical traction test and measurements. For the craniocervical traction test, a tong was attached to the skull under intravenous anesthesia in the supine position and morphometric measurements were taken. Then, the patient was placed in an upright position and craniocervical instability was revealed as neurological symptoms (checked by the third author, R.K.: neurologist), and when morphometric measurements were taken, displacement at the occipitoatlantoaxial joints $(>1$ standard deviation [SD]) was observed. Craniocervical traction of 10 to $15 \mathrm{~kg}$ was then applied and the neurological symptoms resolved (checked by the fourth author, R.K.: neurologist) and a reduction of the craniocervical junction was observed.

Tethered cord syndrome was diagnosed by neurological symptoms (e.g., motor weakness at both lower extremities, sensory loss, neurogenic bladder, pes equinus, and conus medullaris lower than the $\mathrm{L} 2$ vertebral body and/or diameter of the filum terminale $\geq 2 \mathrm{~mm}$ ). ${ }^{15}$ The third author (R.K.: neurologist) diagnosed tethered cord syndrome.

\section{Surgical Indications and Procedures (-Table 3)}

According to the mechanism of ptosis of the brain stem and cerebellum, a surgical procedure was selected. The indications for surgery were the presence of myelopathy, upper cervical cord symptoms, brain stem symptoms, and $<14$

Table 3 Subtypes (CM-I types A, B, and C), surgical indication, and associated anomalies

\begin{tabular}{|c|c|c|c|c|}
\hline Total & 550 cases & $\begin{array}{l}\text { Male/ } \\
\text { female }\end{array}$ & Age & $\begin{array}{l}\text { Mean } \\
\text { (SD) }\end{array}$ \\
\hline & & $255 / 295$ & $16-69$ y & $37.7 \mathrm{y}$ \\
\hline \multicolumn{5}{|l|}{ Subtypes } \\
\hline CM-I type A & 167 cases & $\begin{array}{l}80 / 87 \\
\text { cases }\end{array}$ & $16-69 y$ & $\begin{array}{l}39.6 \mathrm{y} \\
(10.1)\end{array}$ \\
\hline CM-I type B & 178 cases & $\begin{array}{l}83 / 95 \\
\text { cases }\end{array}$ & $17-64$ y & $\begin{array}{l}37.8 \mathrm{y} \\
(10.8)\end{array}$ \\
\hline CM-I type $C$ & 155 cases & $\begin{array}{l}74 / 81 \\
\text { cases }\end{array}$ & $16-59 y$ & $\begin{array}{l}33.4 y \\
(10.3)\end{array}$ \\
\hline CM-absence & 50 cases & $\begin{array}{l}18 / 32 \\
\text { cases }\end{array}$ & $17-55 y$ & $\begin{array}{l}35.4 y \\
(10.4)\end{array}$ \\
\hline Surgery & $\begin{array}{l}585 \\
\text { surgeries }\end{array}$ & & & \\
\hline FMD & 280 cases & $\begin{array}{l}112 / 168 \\
\text { cases }\end{array}$ & $16-69$ y & $\begin{array}{l}37.6 y \\
(10.5)\end{array}$ \\
\hline ESCP & 150 cases & $\begin{array}{l}69 / 81 \\
\text { cases }\end{array}$ & $16-59$ y & $\begin{array}{l}33.4 \mathrm{y} \\
(10.3)\end{array}$ \\
\hline$+\mathrm{OCF}$ & 52 cases & & & \\
\hline CCF & 110 cases & $\begin{array}{l}52 / 58 \\
\text { cases }\end{array}$ & $16-51 y$ & $\begin{array}{l}30.4 \mathrm{y} \\
(10.7)\end{array}$ \\
\hline OCF & 64 cases & & & \\
\hline $\mathrm{C} 1 / 2 \mathrm{PLF}$ & 46 cases & & & \\
\hline SFT & 25 cases & $74 / 81$ & $16-23$ y & $\begin{array}{l}21.8 \mathrm{y} \\
(7.8)\end{array}$ \\
\hline VPS & 20 cases & $\begin{array}{l}8 / 12 \\
\text { cases }\end{array}$ & $16-22$ y & $\begin{array}{l}20.4 y \\
(4.4)\end{array}$ \\
\hline \multicolumn{5}{|c|}{ Associated anomalies } \\
\hline Syringomyelia & $\begin{array}{l}221 \text { cases } \\
(55 \%)\end{array}$ & & & \\
\hline HDCT & $\begin{array}{l}187 \text { cases } \\
(34 \%)\end{array}$ & & & \\
\hline $\begin{array}{l}\text { Basilar } \\
\text { invagination }\end{array}$ & $\begin{array}{l}23 \text { cases } \\
(4.2 \%)\end{array}$ & & & \\
\hline $\begin{array}{l}\text { Other bony } \\
\text { anomalies }\end{array}$ & $\begin{array}{l}57 \text { cases } \\
(10 \%)\end{array}$ & & & \\
\hline
\end{tabular}

Abbreviations: CCF, craniocervical fixation; CM-absence, cases which have neurological brain stem symptoms and/or myelopathy but tonsillar herniation less than $5 \mathrm{~mm}$; CM-I, Chiari malformation type I; ESCP, expansive suboccipital cranioplasty; FMD, foramen magnum decompression, HDCT, hereditary disorders of connective tissue; OCF, occipitocervical fixation; PLF, posterior lateral fixation; SD, standard deviation; SFT, section of the filum terminale; VPS, ventriculoperitoneal shunt. 


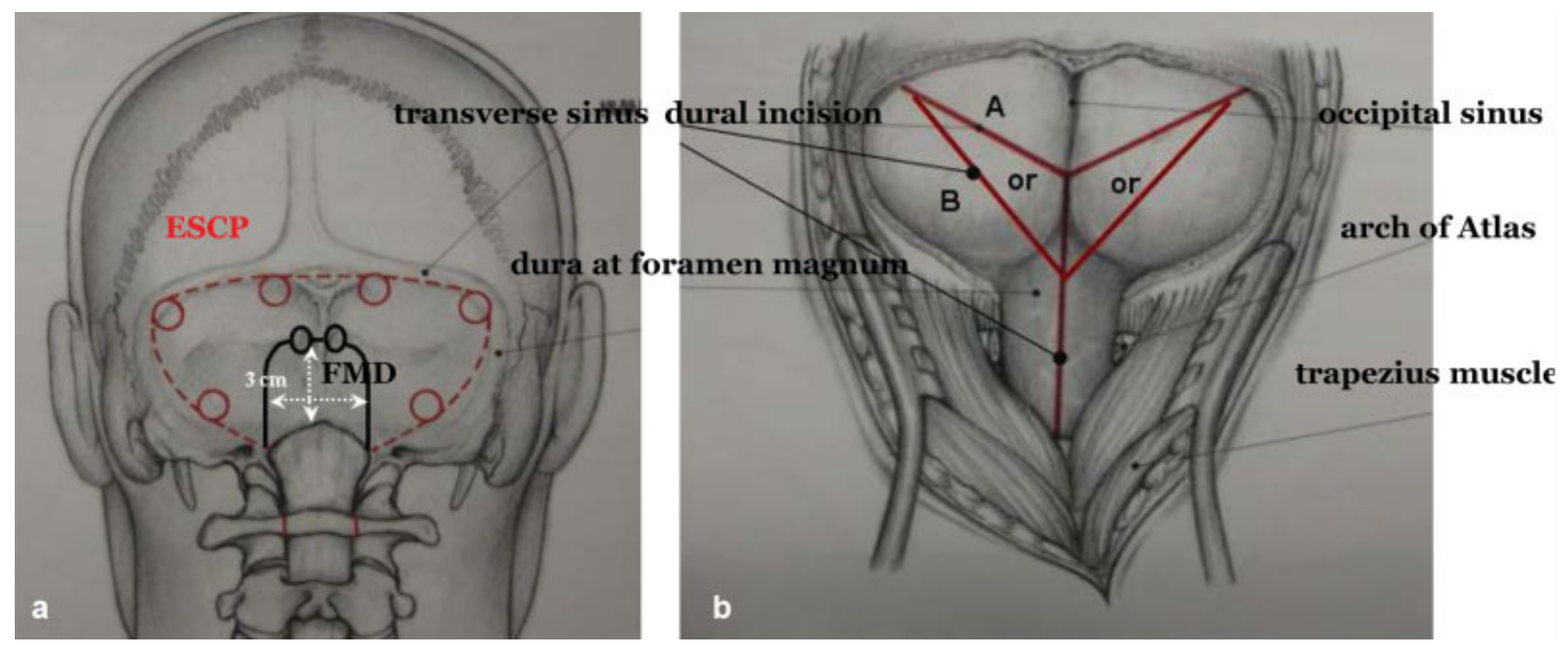

Fig. 1 Craniotomy with expansive suboccipital cranioplasty (ESCP) and foramen magnum decompression (FMD). (Left) Craniotomy with ESCP along the transverse and sigmoid sinuses, and craniotomy with FMD of 2 to $3 \mathrm{~cm}^{2}$. In FMD, the suboccipital muscle group is preserved. (Right) Dural incision in both procedures.

points in the Japanese Orthopedics Association Cervical Myelopathy Evaluation Questionnaire (JOACMEQ). ${ }^{15}$

FMD consists of craniectomy to decompress the surrounding area $\left(2-3 \mathrm{~cm}^{2}\right) .^{16}$ Expansive suboccipital cranioplasty (ESCP), which was described by Sakamoto et al and Nishikawa and Ohata, can also be used for extensive decompression (along the transverse and sigmoid sinuses) and osteoplasty and dural plasty ( - Figs. 1 and $\mathbf{2}$ ). ${ }^{17,18} \mathrm{CM}$-I types A and B (280 cases) underwent FMD to expand the area surrounding the foramen magnum and major cistern and resolve compression of the brain stem and cerebellum (- Figs. 1 and 2 ). ${ }^{15} \mathrm{CM}-\mathrm{I}$ type $\mathrm{C}$ (150 cases) underwent ESCP to expand the area surrounding the foramen magnum and the PCF ( - Figs. 1 and 2). ${ }^{16,17} \mathrm{CM}$ absence (30 cases) underwent FMD. ${ }^{15} \mathrm{C} 1$ laminectomy and dural plasty were performed in all cases. For the cases with instability at the craniocervical junction, posterior craniocervical fixation (CCF) was performed. ${ }^{18}$ In the cases who received ESCP and FMD, CCF was performed on 52 subjects. ${ }^{10-12}$ CCF was used in a total of 110 cases, of which CCF was performed on 64 cases and posterior atlantoaxial fixation was utilized in 46 cases. For tethered cord syndrome, untethering (lysis of the arachnoid adhesion and/or sectioning of the filum terminale [SFT]) was administered to 25 cases. ${ }^{9,13}$ For the cases with increased intracranial pressure and/or hydrocephalus, a ventriculoperitoneal shunt was performed in 20 cases. The other 10 cases had a mass lesion of the PCF, which was removed; 7 cases had an arachnoid cyst, for which fenestration of the cyst was performed.
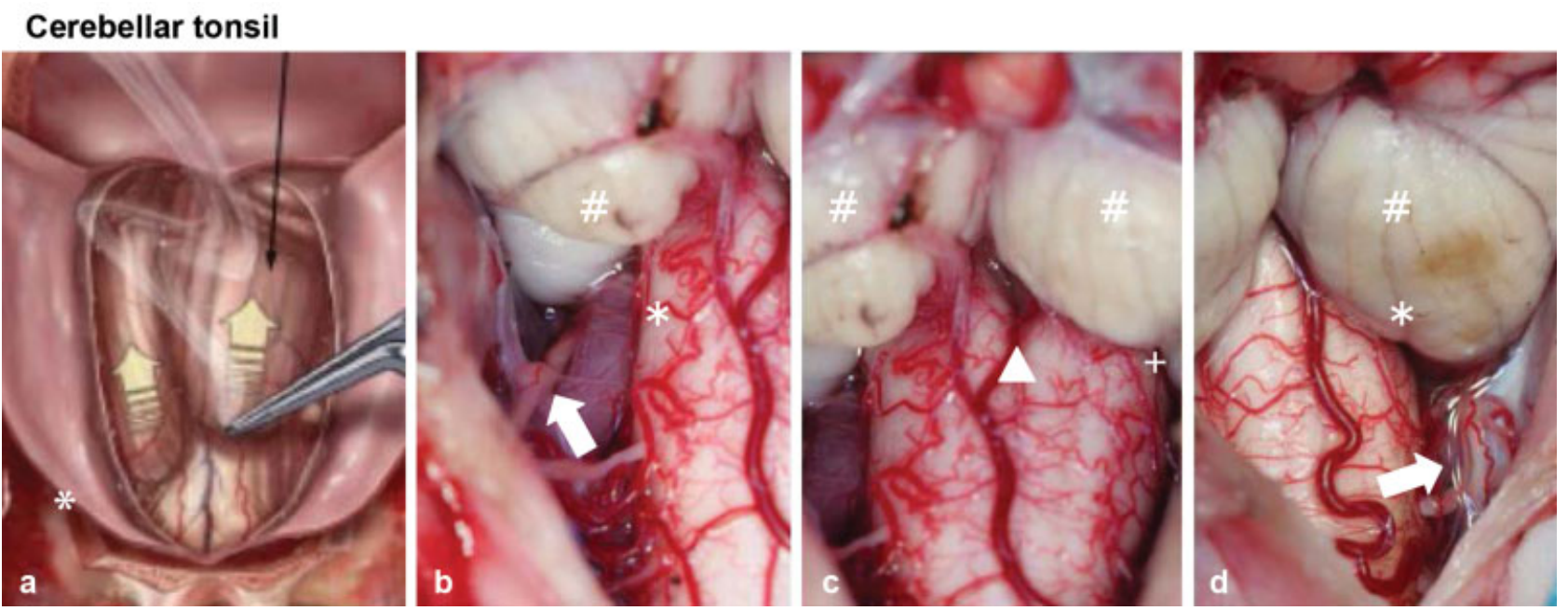

Fig. 2 Operative images, tonsillar burning, and the foramina of Magendie and Luschka. (a) Burning of the cerebellar tonsils. (b) Left foramen of Luschka (*), burned and shrunken tonsil (\#), accessory nerve (arrow), and vertebral artery $(+)$. (c) Foramen of Magendie $\left(^{*}\right)$ ), burned and shrunken tonsils (\#), and obex (arrowhead). (d) Right foramen of Luschka $(*)$, burned and shrunken tonsil (\#), and accessory nerves (arrow). If the tonsils are large and cerebrospinal fluid (CSF) flow is normal after craniotomy and opening the dura mater is not confirmed, the tonsils should be burned to shrink them. Confirmation of CSF flow from the foramina of Magendie and Luschka $\left({ }^{*}\right)$ is important after shrinking the tonsils (\#); CSF flowed from the foramen of Luschka and decompression was performed for accessory nerves (arrows) and the vertebral artery (+), and the obex was observed (arrowhead). 


\section{Examination of Cerebrospinal Fluid Space and Flow}

After bony decompression, before opening the arachnoid membrane and dura mater, and after opening the dura mater and dural plasty, color Doppler ultrasonography (CDU) was performed to observe the dynamics of cerebrospinal fluid (CSF) flow from the foramina of Magendie and Luschka, and the volume of the major cistern and CSF dynamics were estimated (- Fig. 3). ${ }^{19-21}$ Using this approach, Milhorat and Bolognese reported that, as a final goal, the CSF space of the major cistern should be $\geq 8 \mathrm{~mL}$ and the maximum CSF flow velocity should be $\geq 5 \mathrm{~cm} / \mathrm{s} .{ }^{19}$ If the final goal is not achieved, enlargement of the bony decompression should be performed initially; however, if the final goal is still not achieved, burning and shrinking of the cerebellar tonsils should be conducted. ${ }^{19}$

\section{Follow-up and Postoperative Examinations and Determination of Joint Fixation}

Postsurgery, neurological symptoms, activities of daily living (JOACMEQ score), recovery rate of the JOACMEQ score (JOACMEQ score RR), as calculated by (postsurgery points - presurgery points/full points [17] - presurgery points) $\times 100 \%,{ }^{15}$ and neuroradiological findings (dynamic X-ray, 2D CT, and MRI of the cervical spine) were examined every 3 months. Joint fixation was examined to certify that there was no joint instability and/or continuity between bones by cervical spine dynamic X-ray and 2D CT imaging.

\section{Statistical Analysis}

For the comparison of means between two groups, the Mann-Whitney's test was used. For the comparison of more than two groups, the Kruskal-Wallis' test was used. A $p$-value $<0.01$ was used to determine significance. Outcome was assessed using chi-square and Fisher's tests. A pathological condition was defined when VPCF, VSFM, and occipital bone size were $<2$ SD.

The first author (M.N.: neurosurgeon), second author (P. B.: neurosurgeon), third author (R.K.: neurologist), and fourth author (H.I.: neuroradiologist) performed measurements; M.N., P.B., and fifth author (T.T.) performed surgery; M.N. and R.K. performed statistical analyses; and the last author (K.O.) and T.M. conducted and supported this study.

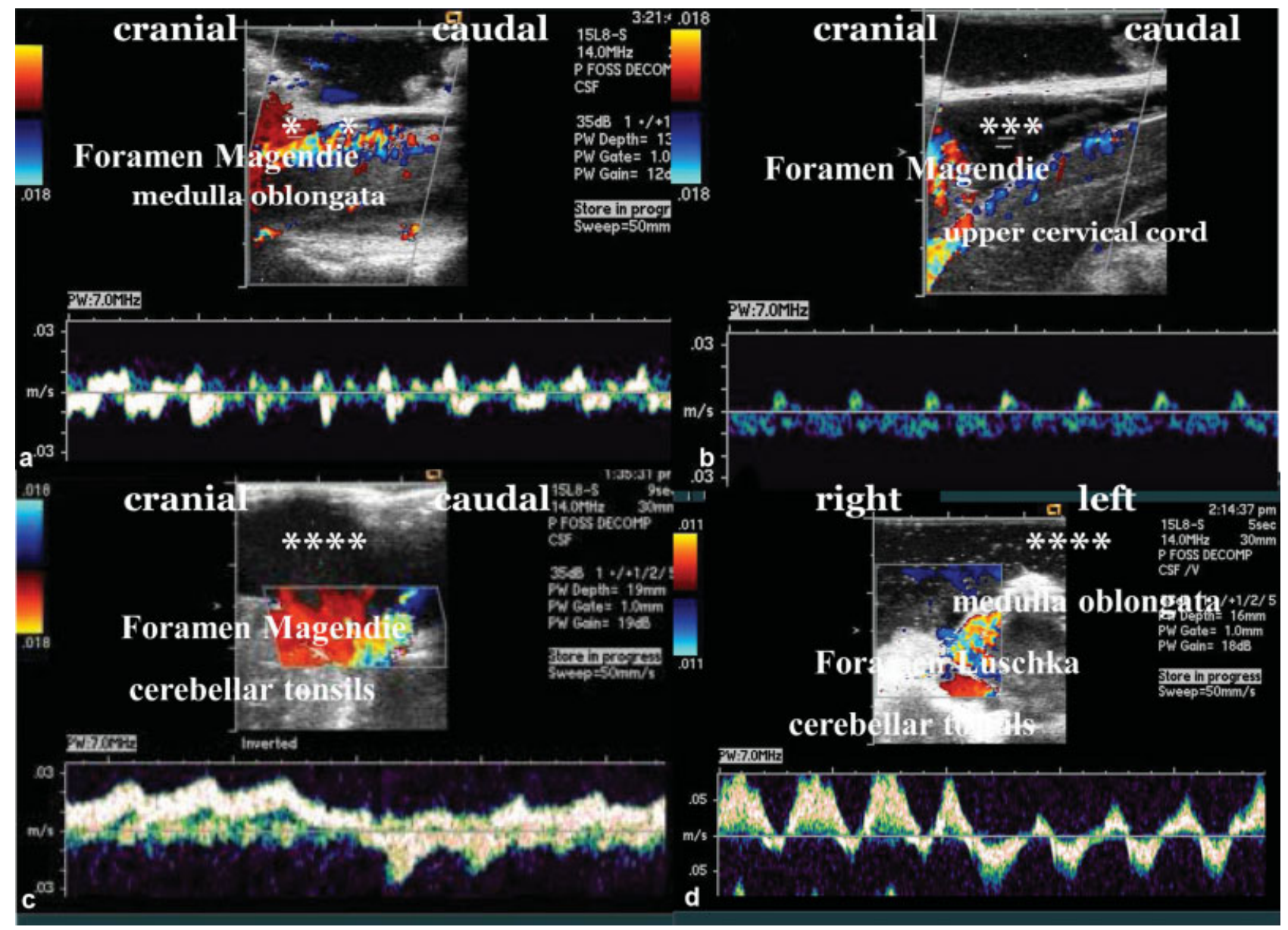

Fig. 3 Color Doppler ultrasonography (CDU). (a) CDU at the foramen of Magendie after craniotomy. The cerebrospinal fluid (CSF) space of the cisterna magna was very small. A small amount of CSF flow was observed. (b) CDU at the foramen of Magendie after cutting the external layer of the dura mater. Although CSF space was slightly increased, there was no change in CSF flow, with only slight flow observed. (c) CDU at the foramen of Magendie after dural plasty. (d) CDU at the foramen of Luschka after dural plasty. In this case, after craniotomy, CDU was performed, and the space of the cisterna magna was found to be small with insufficient CSF flow, and so dural plasty was performed. After dural plasty, CDU was performed again. A large amount of CSF flow from the foramina of Magendie and Luschka was confirmed. Suitable space of the cisterna magna: $12 \mathrm{~mL}$. Maximum CSF flow velocity: $3 \mathrm{~cm} / \mathrm{s}$ at the foramen of Magendie and $10 \mathrm{~cm} / \mathrm{s}$ at the foramen of Luschka. ${ }^{*}$, CSF space. 
Table 4 Overall results: FMD and ESCP

\begin{tabular}{|c|c|c|c|c|}
\hline & Improved & JOACMEQS RR (\%) & Stabilization & Deteriorated \\
\hline FMD: 280 cases (for CM-I types A, B, and CM-absence) & $247(88 \%)^{\mathrm{a}}$ & $66.7 \pm 10.2$ & $25(8.9 \%)$ & $8(2.9 \%)$ \\
\hline Without OAM: 145 & $126(87 \%)^{a}$ & $66.9 \pm 10.5$ & $15(10 \%)$ & \\
\hline With OAM: 135 & $121(90 \%)^{\mathrm{a}}$ & $66.4 \pm 8.7$ & $10(7.4 \%)$ & \\
\hline ESCP 150 cases (for CM-type C) & $131(87 \%)^{\mathrm{a}}$ & $66.7 \pm 10.1$ & $16(11 \%)$ & $3(2.0 \%)$ \\
\hline Without OAM: 72 & $63(88 \%)^{a}$ & $70.5 \pm 9.6$ & $8(11 \%)$ & $1(1.4 \%)$ \\
\hline With OAM: 78 & $68(87 \%)^{a}$ & $64.5 \pm 10.7$ & $8(11 \%)$ & $2(2.6 \%)$ \\
\hline \multicolumn{2}{|l|}{ Complication: wound infection: $3(1.1 \%)$ in FMD } & & & \\
\hline \multicolumn{3}{|c|}{ Wound infection: 2 (1.3\%) and cerebellar slumping: $10.7 \%)$ in ESCP } & & \\
\hline
\end{tabular}

Abbreviations: CM-absence, cases which have neurological brain stem and/or myelopathy but tonsillar herniation less than $5 \mathrm{~mm}$; CM-I, Chiari malformation type I; ESCP, expansive suboccipital cranioplasty; FMD, foramen magnum decompression; JOACMEQS RR, recovery rate of Japanese Orthopedics Association Cervical Myelopathy Evaluation Questionnaire Score; OAM, opening of arachnoid membrane.

Note: Follow-up period: 18 to 130 months, mean: 40.5 months.

a Significantly high $(p<0.001)$.

\section{Results}

\section{Outcome of Surgical Treatment}

The follow-up period was 18 to 130 months (mean: 40.5 months). Outcome was estimated using the most recent data. Twenty-eight cases dropped out of the study at more than 3 years after surgery, in whom the results of the final examination were estimated. Only 12 cases were missed during follow-up.

FMD was performed in cases with CM-I type A, CM-I type B, and CM-absence. The JOACMEQ score RR for FMD was 66.7\%, while $97 \%$ of cases showed an improvement or stabilization of their neurological symptoms. There was no significant difference in the JOACMEQ score RR between the cases in which the arachnoid membrane was or was not opened. In eight cases (2.9\%), their neurological symptoms deteriorated later (-Table 4). In 7 of 207 cases, syringomyelia remained.

ESCP was performed for CM-I type C. The JOACMEQ score RR of ESCP was $66.7 \%$, and $98 \%$ of cases had an improvement or stabilization of their neurological symptoms. There was no significant difference in the JOACMEQ score RR between the cases in which the arachnoid membrane was or was not opened. In three cases (2.0\%), their neurological symptoms deteriorated later. In all 110 cases, syringomyelia vanished.

There was no significant difference between the FMD and ESCP groups for the improvement or stabilization of neurological symptoms and the JOACMEQ score RR. Complications were observed in three cases (1.1\%) with FMD, and two cases (1.3\%) with ESCP had a wound infection. In MRI, cerebellar slugging without neurological symptoms occurred with ESCP. In both the FMD and ESCP groups, there was no mortality and no permanent morbidity (-Table 4 ).

Of the cases who underwent CCF, 97 (88\%) had an improvement of their neurological symptoms and the JOACMEQ score RR was $76.9 \%$. Sixty-eight cases (62\%) had complete bony fusion, while 33 cases (30\%) were stabilized. Nine cases (8\%) still had incomplete stabilization. Complications consisted of a transient swallowing disturbance in one case ( $0.9 \%)$, injury to the vertebral artery without neurological symptoms in one case $(0.9 \%)$, and wound infection in one case $(0.9 \%)$. There was no permanent morbidity (-Table $\mathbf{5}$ ).

Untethering was performed in 25 cases with tethered cord syndrome; 9 cases (36\%) demonstrated an improvement of their neurological symptoms, while the other 16 cases (64\%)

Table 5 Overall results: CCF

\begin{tabular}{|l|l|l|l|}
\hline Clinical symptoms & Improved & JOACMEQS RR (\%) & Unchanged \\
\hline 110 cases & $97(88 \%)$ & $76.9 \pm 13.2$ & $13(12 \%)$ \\
\hline Fixation and stabilization of joints & Complete fusion & Stabilization & Incomplete \\
\hline 110 cases & $68(62 \%)$ & $33(30 \%)$ & $9(8 \%)$ \\
\hline Complication: transient swallowing disturbance: $1(0.9 \%)$ & & \\
\hline Wound infection: $1(0.9 \%)$ & & \\
\hline Vertebral artery injury: 1 (0.9\%) & & \\
\hline Permanent complication: none & Morbidity: 0 & \\
\hline
\end{tabular}

Abbreviations: CCF, craniocervical fixation; JOACMEQS RR, recovery rate of Japanese Orthopedics Association, Cervical Myelopathy Evaluation Questionnaire Score.

Note: Follow-up period: 18 to 130 months, mean: 40.5 months. 
had no neurological improvement, but rather deteriorated, and so FMD was added and their neurological symptoms improved. In the cases with increased intracranial pressure and/or hydrocephalus, a ventriculoperitoneal shunt was performed in 20 cases; 10 cases (50\%) demonstrated an improvement of their neurological symptoms. The other 10 cases (50\%) had no neurological improvement, so FMD was added and their neurological symptoms improved.

\section{Reoperation Cases (-Table 6)}

There were 22 cases (5.1\%) who had deterioration of their neurological symptoms and/or the syrinx was not reduced: CM-I type A, 11 cases (8.5\%); CM-I type B, 8 cases (6.0\%); CM-I type C, 1 case (0.7\%); and CM-absence: 2 cases (6.7\%). There were significantly more of these cases in the CM-I type A, CM-I type $\mathrm{B}$, and $\mathrm{CM}$-absence groups than in the $\mathrm{CM}-\mathrm{I}$ type $\mathrm{C}$ group. The most common reason for a second operation was instability at the CVJ in 12 cases (55\%), with 11 of these cases in the CM-I type A, CM-I type B, and CM-absence groups, while inappropriate decompression was the cause in 7 cases (32\%) in the CM-I type A and B groups. Two cases (9\%) in the CM-I type A group developed tethered cord syndrome and SFT was added. One case $(4.5 \%)$ in the CM-I type B group developed an arachnoid adhesion due to blood flow into the subarachnoid space during the initial operation, and lysis of the adhesion and untethering were performed. Only one case of cerebellar slumping (downward displacement of the cerebellum into the enlarged major cistern and displacement of the brain stem and cerebellar hemisphere) was observed in the ESCP group.

\section{Discussion}

Mechanisms: Pathogenesis of Ptosis of the Brain Stem and Cerebellum, and Surgical Indication and Outcome in CM-I type B, CM-I type C, and CM-absence

In these cases, ptosis of the brain stem and cerebellum was caused by a small VSFM and underdevelopment of the occipital bone. From the embryological viewpoint, the occipital bone is formed from enchondral bone that originates from occipital somites. Therefore, the pathogenesis of CM-I type B, CM-I type C, and CM-absence was insufficiency of the para-axial mesoderm, as the source of the occipital bone., ${ }^{1,2}$

In the management of Chiari malformation, appropriate surgical methods that can treat ptosis of the brain stem and cerebellum should be chosen. On the basis of this idea, we selected FMD for CM-I type B and CM-absence because VSFM and occipital bone size were small. ${ }^{15}$ We chose ESCP for CM-I type $C$ because FMD was not considered appropriate under the setting of a small VSFM, small VPCF, and small occipital bone size. ${ }^{16}$ ESCP can normalize CSF flow, but it is also possible that the enlarged space created by ESCP could result in displacement of the brain stem and cerebellum into the PCF.

Both ESCP and FMD had good surgical outcomes. These outcomes were better than those reported previously in cases receiving only FMD, and morbidity and complications were also less than in previous reports. ${ }^{16,17,19,22-26}$ This suggested that the choice of ESCP or FMD according to classification by morphometric analyses was appropriate.

\section{Mechanisms of Ptosis of the Brain Stem and Cerebellum, and Surgical Indication in CM-I type A}

In CM-I type A, which has normal VPCF, normal VSFM, and normal occipital bone size, there was no significant difference in the VPCB/VPCF ratio compared with the normal control group. Therefore, the mechanism underlying ptosis of the brain stem and cerebellum was not the narrowness of the PCF, but due to other mechanisms. Milhorat et al and the authors of the present study have reported the phenomenon of functional cranial settling, in which the cranium (occipital bone) falls into the CVJ. This functional cranial settling could cause ptosis of the brain stem and cerebellum. ${ }^{8}$ In addition, Milhorat et al and the authors of the present study have indicated that tethering could cause ptosis of the brain stem and cerebellum by a traction effect. ${ }^{9}$

In CM-I type A, various mechanisms could cause ptosis of the brain stem and cerebellum. Other surgical methods that can treat ptosis of the brain stem and cerebellum must be chosen. CCF should be chosen for cases with craniocervical instability. Untethering and/or SFT should be chosen for cases with a lesion of traction and/or tethering.

Table 6 Reoperation cases

\begin{tabular}{|l|l|l|l|l|}
\hline \multirow{2}{*}{} & CM-I type A & CM-I type B & CM-I type C & CM-absence \\
\cline { 2 - 5 } & FMD & FMD & ESCP & FMD \\
\hline $5.1 \%$ & $11 / 117$ & $8 / 133$ & $1 / 150$ & $2 / 30(6.7 \%)^{\mathrm{a}}$ \\
\hline Other mechanism of ptosis of the brain stem and cerebellum & $6.0 \%^{\mathrm{a}}$ & $0.7 \%^{\mathrm{a}}$ & $6.7 \%^{\mathrm{a}}$ \\
\hline CV instability & $8.5 \%^{\mathrm{a}}$ & 4 & 1 & 2 \\
\hline Incomplete decompression & 5 & 3 & & \\
\hline Tethered cord & 4 & & & \\
\hline Arachnoid adhesion & 2 & 1 & & \\
\hline
\end{tabular}

Abbreviations: CM-absence, cases which have neurological brain stem symptom and/or myelopathy but tonsillar herniation less than $5 \mathrm{~mm}$; CM-I, Chiari malformation type I; CVJ, craniovertebral junction; ESCP, expansive suboccipital cranioplasty; FMD, foramen magnum decompression. Note: Follow-up period: 18 to 130 months, mean: 40.5 months.

${ }^{\text {aS }}$ ignificantly high compared with CM-I type C group $(p<0.001)$. 
In five cases who needed CCF after FMD or ESCP, CCF using the diploic screw technique, which we have described previously, was effective. $^{27}$

\section{Color Doppler Ultrasonography}

The examination of CSF flow using CDU was very important. ${ }^{19}$ In the present study, there was no difference in outcome between opening or not opening the arachnoid membrane. We were not concerned about preserving the arachnoid membrane at the major cistern, and confirming that CSF flowed out from the foramina of Magendie and Luschka was much more important. In all cases, we examined the dynamics of CSF flow at the major cistern using CDU (-Fig. $\mathbf{3}$ ). At the point of bony decompression, the CSF flow dynamics data never reached their previous levels. In the early stage (ESCP: five cases; FMD: five cases) at the point of incision of the outer membrane, CSF flow dynamics never reached their previous levels; therefore, dural plasty was added to all cases. Then, only outer membrane resection was not performed.

\section{Preliminary Results of CCF}

CCF led in a significant improvement of neurological symptoms and had a high rate of joint fixation. These results suggested that CCF was an effective surgical approach for cranial settling due to craniovertebral hypermobility or instability (-Table 5). To investigate hypermobility and instability at the occipitoatlantoaxial joints, a craniocervical traction test should be performed. ${ }^{8}$

\section{Preliminary Results of Lysis, SFT, and Ventriculoperitoneal Shunt}

Less than half of the cases with both untethering (lysis and/ or SFT) and a ventriculoperitoneal shunt demonstrated an improvement of their neurological symptoms. Most of these cases were followed by FMD/ESCP and other surgical procedures, leading to an improvement of their symptoms. Therefore, only lysis and/or SFT and a ventriculoperitoneal shunt are not fundamental treatments for cases with CM-I and CM-absence (-Table 5).

\section{Reoperation Cases (-Table 6)}

Craniocervical instability was the most common reason for reoperation, and was observed significantly more often in CM-I type A, CM-I type B, and CM-absence than in CM-I type C.

Cases with CM-I type A, CM-I type B, and CM-absence should undergo a craniocervical traction test and examination of hypermobility and instability at the occipitoatlantoaxial joints. CCF had a good surgical outcome with regard to the improvement of neurological symptoms and fixation. In cases with CM-I types $\mathrm{A}$ and $\mathrm{B}$, the FMD examination should include CDU to measure CSF flow and decompression of the brain stem and cerebellum.

\section{Conclusion}

Morphometric analyses of the PCF and CVJ are required to determine the mechanism and treatment of ptosis of the brain stem and cerebellum.
According to the mechanism of ptosis of the brain stem and cerebellum in the three types of CM-I, which were suggested by VPCF and morphometric analyses, a surgical procedure was selected. Each surgical treatment resulted in a good improvement of symptoms and safety course.

In the management of Chiari malformation, appropriate surgical methods that address ptosis of the brain stem and cerebellum should be chosen. It is important to perform appropriate decompression, while examination of CSF dynamics using CDU during surgery is recommended.

Note

This study has been approved by Institutional Review Boards of Koudoukai Health System, Osaka City University Graduate School of Medicine, Osaka, Japan, and North Shore University Hospital-Long Island Jewish Health System, New York, United States.

\section{Conflict of Interest}

None declared.

\section{Acknowledgments}

The authors deeply thank Professor Emeritus of North Shore University Hospital-Long Island Jewish Health System, Thomas H. Milhorat, MD, for leading and supporting this study.

\section{References}

1 Nishikawa M, Sakamoto H, Hakuba A, Nakanishi N, Inoue Y. Pathogenesis of Chiari malformation: a morphometric study of the posterior cranial fossa. J Neurosurg 1997;86(01):40-47

2 Milhorat TH, Chou MW, Trinidad EM, et al. Chiari I malformation redefined: clinical and radiographic findings for 364 symptomatic patients. Neurosurgery 1999;44(05):1005-1017

3 Alperin N, Loftus JR, Oliu CJ, et al. Magnetic resonance imaging measures of posterior cranial fossa morphology and cerebrospinal fluid physiology in Chiari malformation type I. Neurosurgery 2014;75(05):515-522, discussion 522

4 Badie B, Mendoza D, Batzdorf U. Posterior fossa volume and response to suboccipital decompression in patients with Chiari I malformation. Neurosurgery 1995;37(02):214-218

5 Cinalli G, Spennato P, Sainte-Rose C, et al. Chiari malformation in craniosynostosis. Childs Nerv Syst 2005;21(10):889-901

6 Karagöz F, Izgi N, Kapíjcíjoğlu Sencer S. Morphometric measurements of the cranium in patients with Chiari type I malformation and comparison with the normal population. Acta Neurochir (Wien) 2002;144(02):165-171, discussion 171

7 Stovner LJ, Bergan U, Nilsen G, Sjaastad O. Posterior cranial fossa dimensions in the Chiari I malformation: relation to pathogenesis and clinical presentation. Neuroradiology 1993;35(02):113-118

8 Milhorat TH, Bolognese PA, Nishikawa M, McDonnell NB, Francomano CA. Syndrome of occipitoatlantoaxial hypermobility, cranial settling, and Chiari malformation type I in patients with hereditary disorders of connective tissue. JNeurosurg Spine 2007;7(06):601-609

9 Milhorat TH, Bolognese PA, Nishikawa M, et al. Association of Chiari malformation type I and tethered cord syndrome: preliminary results of sectioning filum terminale. Surg Neurol 2009;72 (01):20-35

10 Goel A, Jain S, Shah A. Radiological evaluation of 510 cases of basilar invagination with evidence of atlantoaxial instability (group a basilar invagination). World Neurosurg 2018;110:533-543 
11 Goel A, Gore S, Shah A, Dharurkar P, Vutha R, Patil A. Atlantoaxial fixation for Chiari 1 formation in pediatric age group patients: report of treatment in 33 patients. World Neurosurg 2018;111:e668-e677

12 Goel A, Kaswa A, Shah A. Atlantoaxial fixation for treatment of Chiari formation and syringomyelia with no craniovertebral bone anomaly: report an experience with 57 cases. Acta Neurochir Suppl (Wien) 2018;125:101-110

13 Milhorat TH, Nishikawa M, Kula RW, Dlugacz YD. Mechanisms of cerebellar tonsil herniation in patients with Chiari malformations as guide to clinical management. Acta Neurochir (Wien) 2010;152 (07):1117-1127

14 Nishikawa M, Bolognese PA, Kula RW, et al. Pathogenesis and classification of Chiari malformation type I based on the mechanism of ptosis of the brain stem and cerebellum: a morphometric study of the posterior cranial fossa and craniovertebral junction. J Neurol Surg B Skull Base, in press

15 Fukui M, Chiba K, Kawakami M, et al; Subcommittee on Low Back Pain and Cervical Myelopathy Evaluation of the Clinical Outcome Committee of the Japanese Orthopaedic Association. An outcome measure for patients with cervical myelopathy: Japanese Orthopaedic Association Cervical Myelopathy Evaluation Questionnaire (JOACMEQ): Part 1. JOrthop Sci 2007;12(03):227-240

16 Oaks J. Chiari malformation and syringomyelia. In: Reagachary SS, Williams RH, eds. Principles of Neurosurgery. London: MosbyWolf; 1994:11-18

17 Sakamoto H, Nishikawa M, Hakuba A, et al. Expansive suboccipital cranioplasty for the treatment of syringomyelia associated with Chiari malformation. Acta Neurochir (Wien) 1999;141 (09):949-960, discussion 960-961

18 Nishikawa M, Ohata K. Surgical treatment of Chiari malformation. In: Ohata K, ed. Spinalcord Surgery Neurosurgery Now. Tokyo: Medical View; 2011:138-153
19 Milhorat TH, Bolognese PA. Tailored operative technique for Chiari type I malformation using intraoperative color Doppler ultrasonography. Neurosurgery 2003;53(04):899-905, discussion 905-906

20 Cui LG, Jiang L, Zhang HB, et al. Monitoring of cerebrospinal fluid flow by intraoperative ultrasound in patients with Chiari I malformation. Clin Neurol Neurosurg 2011;113(03):173-176

21 Bond AE, Jane JA Sr, Liu KC, Oldfield EH. Changes in cerebrospinal fluid flow assessed using intraoperative MRI during posterior fossa decompression for Chiari malformation. JNeurosurg 2015; 122(05):1068-1075

22 Winegar CD, Lawrence JP, Friel BC, et al. A systematic review of occipital cervical fusion: techniques and outcomes. J Neurosurg Spine 2010;13(01):5-16

23 Batzdorf U, McArthur DL, Bentson JR. Surgical treatment of Chiari malformation with and without syringomyelia: experience with 177 adult patients. J Neurosurg 2013;118(02):232-242

24 Klekamp J, Batzdorf U, Samii M, Bothe HW. The surgical treatment of Chiari I malformation. Acta Neurochir (Wien) 1996;138(07): 788-801

25 Tubbs RS, Beckman J, Naftel RP, et al. Institutional experience with 500 cases of surgically treated pediatric patients with Chiari malformations. J Neurosurg Pediatr 2011;7:248-256

26 Arnautovic A, Splavski B, Boop FA, Arnautovic KI. Pediatric and adult Chiari malformation Type I surgical series 1965-2013: a review of demographics, operative treatment, and outcomes. J Neurosurg Pediatr 2015;15(02):161-177

27 Nishikawa M, Ohata K, Baba M, Terakawa Y, Hara M. Chiari I malformation associated with ventral compression and instability: one-stage posterior decompression and fusion with a new instrumentation technique. Neurosurgery 2004;54(06):1430-1434, discussion 1434-1435 